\title{
Wald und Forstwirtschaft
}

Michael Köhl, Daniel Plugge, Martin Gutsch, Petra Lasch-Born, Michael Müller, Christopher Reyer

19.1 Wälder im globalen Kohlenstoffkreislauf - 194

19.2 Was der Klimawandel mit dem deutschen Wald macht - 195

19.2.1 Veränderte Ausbreitungsgebiete und Artenzusammensetzung - 195

19.2.2 Längere Vegetationsperioden - 196

19.2.3 Waldschäden: keine einfachen Antworten - 196

19.2.4 Temperatur und Niederschläge beeinflussen Produktivität - 198

19.2.5 Kohlenstoffhaushalt: von der Senke zur Quelle - 198

19.3 Anpassung in der Forstwirtschaft - 198

$19.4 \quad$ Kurz gesagt -199

Literatur - 200 
Fast ein Drittel von Deutschland ist mit Wald bedeckt. Das entspricht etwa 11,4 Mio. Hektar (BMEL 2014). Auf einem Hektar Waldboden stehen durchschnittlich rund $336 \mathrm{~m}^{3} \mathrm{Holz}$ - so viel wie der Inhalt von knapp fünf 40-Fuß-Containern. Mit insgesamt 3662 Mio. $\mathrm{m}^{3}$ besitzt Deutschland den größten Holzvorrat in Europa (Forest Europe 2011; BMEL 2014). Jedes Jahr kommen $11,1 \mathrm{~m}^{3} /$ ha dazu (Oehmichen et al. 2011); Holzeinschlag und natürlicher Abgang (Mortalität) schöpfen jedoch rund $87 \%$ des Zuwachses ab (BMEL 2014).

Durch Fotosynthese und Biomassewachstum entziehen Wälder der Atmosphäre Kohlendioxid $\left(\mathrm{CO}_{2}\right)$ und binden es als Kohlenstoff im Holz. In jedem Kubikmeter Holz stecken je nach Baumart bzw. Holzdichte rund $270 \mathrm{~kg}$ Kohlenstoff. Damit ist der Wald ein wichtiger Kohlenstoffspeicher. Zwischen 2002 und 2012 hat der deutsche Wald jährlich rund 52 Mio. $\mathrm{CO}_{2}$ aus der Atmosphäre aufgenommen (Dunger et al. 2014) und wirkt deshalb in diesem Zeitraum als Kohlenstoffsenke. Diese Menge entspricht etwa $15 \%$ der durchschnittlichen jährlichen $\mathrm{CO}_{2}$-Emissionen der privaten Haushalte hierzulande.

Wälder produzieren nicht nur den nachwachsenden Rohstoff Holz, sondern sie leisten auch viel für die Umwelt und wirken ausgleichend auf das Klima. Über ihre Blätter und Nadeln verdunsten Bäume Wasser, das sie mit ihren Wurzeln aus dem Boden saugen. Ein Buchenwald kann im Sommer täglich mehrere tausend Liter Wasser pro Hektar verdunsten (Schreck et al. 2016). Der Wasserdampf kondensiert und bildet Wolken; diese reflektieren Sonnenstrahlen und wirken somit der globalen Erwärmung entgegen. Besonders stark ist dieser Effekt in den Tropen.

Klimawandel und Wälder stehen in einem komplexen Wirkungsgefüge: Die Waldzerstörung, vor allem in den Tropen, trägt etwa ein Sechstel zu den jährlichen globalen Treibhausgasemissionen bei; Klimaveränderungen beeinflussen die Produktivität und Lebenskraft (Vitalität) von Wäldern. Energetische und stoffliche Verwendung von geerntetem Holz können den Verbrauch und damit die Emissionen fossiler Energieträger vermindern. Zudem wird Kohlenstoff in der Biomasse, im Waldboden und in Holzprodukten gebunden (FAO 2010; Knauf et al. 2015).

Wälder bedecken ein Drittel der Landfläche der Erde (FAO 2010) und sind der größte Kohlenstoffspeicher auf dem Land (Pan et al. 2011). Eingeteilt werden sie in drei Großlebensräume: nördliche (boreale), gemäßigte und tropische Wälder. Die tropischen Wälder besitzen mit 471 933 Pg (Petagramm) - ein Petagramm entspricht $1 \mathrm{Mrd}$. Tonnen - in ihrer Vegetation und im Boden den größten Kohlenstoffvorrat, gefolgt von den borealen Wäldern mit $272 \pm 23$ Pg Kohlenstoff, der größtenteils im Boden gespeichert ist. In den gemäßigten Breiten sind 119 \pm 9 Pg Kohlenstoff in den Wäldern gespeichert (Pan et al. 2011). Die europäischen Wälder gehören weitgehend der gemäßigten Zone an. Der deutsche Wald speichert 2,2 Pg Kohlenstoff, davon 59\% in der Baumbiomasse, $1 \%$ in Totholz und $40 \%$ in Streu und Waldboden. Pro Jahr nahm der Wald im Bezugszeitraum 1990-2002 22 Mio. t Kohlenstoff auf (BMELV 2009).

\subsection{Wälder im globalen Kohlenstoffkreislauf}

Bäume binden in ihrer Biomasse atmosphärisches $\mathrm{CO}_{2}$ als Kohlenstoff. In Totholz oder Streu wird Kohlenstoff dagegen abgebaut und entweder als $\mathrm{CO}_{2}$ in die Atmosphäre freigesetzt oder als Bodenkohlenstoff aufgenommen. Ist die C-Bindung durch Fotosynthese und Wachstum größer als die $\mathrm{CO}_{2}$-Freisetzung durch Abbauprozesse, wird der Wald zur Kohlenstoffsenke. In Naturwäldern stellt sich über längere Zeit und große Gebiete ein Gleichgewicht zwischen Auf- und Abbau von Biomasse ein (Lal 2005; Luyssaert et al. 2008), sodass sich langfristig betrachtet Bindung und Freisetzung die Waage halten.

Der Nationale Inventarbericht zu Treibhausgasemissionen (NIR) (UBA 2011) und die Ergebnisse der 2. Bodenzustandserhebung zeigen, dass die Kohlenstoffvorräte in den deutschen Waldböden in etwa stabil geblieben oder sogar gestiegen sind (Block und Gauer 2012; Russ und Riek 2011).

Störungen des Waldgefüges, etwa durch Sturmschäden oder Waldsterben nach Insektenbefall, können bewirken, dass über einen längeren Zeitraum große Mengen $\mathrm{CO}_{2}$ in die Atmosphäre gelangen. Neben diesen Schädigungen wird der Wald zu einer $\mathrm{CO}_{2}$-Quelle (Kurz et al. 2008), wenn Wald- und Bodenspeicher durch anthropogen getriebene Entwaldung und Degradation vernichtet werden. Besonders in den Tropen tragen diese Prozesse jährlich mit rund $10-17 \%$ oder 2,8 \pm 0,4 Pg Kohlenstoff zu den globalen Treibhausgasemissionen bei (Houghton 2013; IPCC 2007; Settele et al. 2014). Demgegenüber steht eine Kohlenstoffaufnahme von 4,0 \pm ,7 Pg pro Jahr. Entsprechend kann von einer Senkenfunktion der globalen Wälder von 1,1 $\pm 0,8$ Pg Kohlenstoff pro Jahr ausgegangen werden (Settele et al. 2014).

Bis auf wenige Ausnahmen wie Nationalparks oder Bannwälder werden Deutschlands Wälder bewirtschaftet: Zwischen 2002 und 2008 wurden jährlich etwa 70 Mio. $\mathrm{m}^{3}$ verwertbares Nutzholz geerntet (Oehmichen et al. 2011). Im Kohlenstoffkreislauf spielen Holzernte und -verwendung daher eine wichtige Rolle. Je nach Nutzungsdauer, möglicher Mehrfachnutzung und Verwendungszweck können viele Holzprodukte zum Klimaschutz beitragen:

- In Holz festgelegter Kohlenstoff wird nach der Holzernte in Holzprodukten gespeichert (Produktspeicher).

- Energetische Nutzung von Holz setzt zwar $\mathrm{CO}_{2}$ frei, vermeidet aber gleichzeitig $\mathrm{CO}_{2}$-Emissionen aus fossilen Energieträgern (energetische Substitution).

- Bei der Herstellung funktionsgleicher Produkte verbraucht die Verwendung von Holz in der Regel weniger Energie als die Verwendung alternativer Materialien (z. B. Ziegel, Kalksandsteine, Stahl, Aluminium). Damit lassen sich ebenso Emissionen aus fossilen Energieträgern einsparen (stoffliche Substitution) (Bergman et al. 2014 ; Knauf et al. 2015).

Wälder, die für die energetische Substitution durch Holz genutzt werden, schöpfen nicht das maximale Potenzial der Kohlenstoffspeicherung aus. Darüber hinaus ist die Energiedichte von Holz geringer als die fossiler Energieträger. Hierdurch entsteht eine sogenannte Kohlenstoffschuld. Diese gleicht sich jedoch über einen längeren Zeitraum durch die erhöhten Substitutionseffekte und die begrenzte maximale Kohlenstoffspeicherfähigkeit von Wäldern aus bzw. wird überkompensiert (Mitchell et al. 2012). 


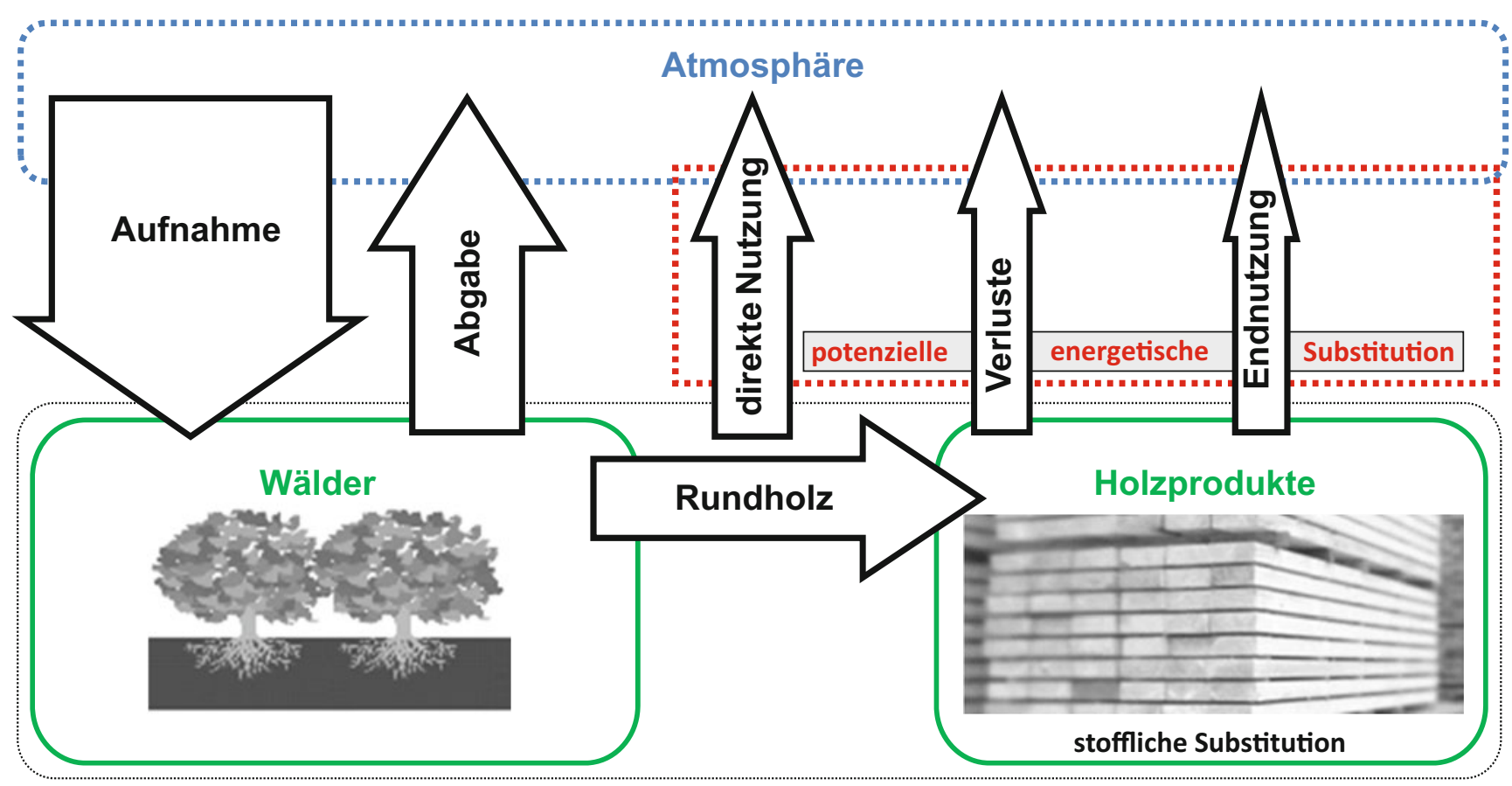

- Abb. 19.1 Wald und Klima - Kohlenstoffspeicher und Kohlenstoffflüsse. Die Größe der Pfeile ist proportional zu den Anteilen an den Kohlenstoffflüssen

Der Waldspeicher vergrößert sich nur so lange, bis ein konstantes Gleichgewicht zwischen Auf- und Abbau von Biomasse erreicht ist. Dagegen akkumulieren die positiven Effekte der Substitution mit der Zeit, es wird ein „Vermeidungsguthaben“ aufgebaut. Waldwachstum und stoffliche Holzverwendung verlagern den Kohlenstoff zwischen Atmosphäre, Waldspeicher und Holzproduktspeicher (- Abb. 19.1), aber bringen kein zusätzliches $\mathrm{CO}_{2}$ in dieses System. Der Aufbau von Wald- und Produktspeicher kann, bei entsprechender langfristiger Nutzung der Produkte, einen Nettoeffekt auf die Kohlenstoffbindung haben. Die aktuell wieder populärer werdende Holzverbrennung zur Energiegewinnung führt im Gegensatz zur Verbrennung fossiler Energieträger nicht zu einer Erhöhung des $\mathrm{CO}_{2}$-Gehalts der Atmosphäre.

\subsection{Was der Klimawandel mit dem deutschen Wald macht}

Die erwarteten bzw. projizierten Klimaänderungen im 21. Jahrhundert werden die Zusammensetzung der Baumarten in Deutschlands Wäldern beeinflussen. Auch Schadfaktoren werden sich verändern (Lindner et al. 2010; Müller 2009). Das zieht nicht nur ökologische Folgen nach sich, sondern auch bedeutende ökonomische (Hanewinkel et al. 2012). Wälder waren aufgrund des genetischen Potenzials von Bäumen in der Lage, sich an vergangene Phasen von natürlichem Klimawandel anzupassen. Die aktuelle, teilweise anthropogen bedingte Klimaveränderung weist eine höhere Geschwindigkeit auf als vergleichbare historische Änderungen im Klimasystem. Aufgrund der Langlebigkeit von Bäumen ist ein Abwarten auf die natürliche Anpassung mit den gesellschaftlichen Anforderungen an den Wald als Ökosystem und Ressource voraussichtlich nicht vereinbar. Ein gerichtetes Einschreiten der Forstwirtschaft zum Erhalt der multifunktionalen Wälder ist dementsprechend notwendig. Im Folgenden werden die vorhandenen Erkenntnisse zum Einfluss des Klimawandels auf den deutschen Wald als Grundlage für eine solche Anpassung dargestellt.

\subsubsection{Veränderte Ausbreitungsgebiete und Artenzusammensetzung}

Mithilfe von „Klimahüllenmodellen“ entwickelte der Forstwissenschaftler Christian Kölling 2007 ein erstes Konzept, um die ökologischen Effekte des Klimawandels auf die Anbaumöglichkeiten der Baumarten in Deutschland zu untersuchen (Kölling 2007). Er beschrieb die Häufigkeitsverteilung der wichtigsten Baumarten in Deutschland abhängig von Jahresmitteltemperatur und Jahresniederschlagssumme. Unter Verwendung von Klimaprojektionen für Deutschland von einem Regionalmodell (WETTREG) und einem Emissionsszenario (SRES B1) leitete er Aussagen etwa zur Anbaueignung der Rotbuche (Fagus sylvatica) und Gemeinen Fichte (Picea abies) ab: In Bayern könnte die Rotbuche demnach zusätzlich höhere Gebirgslagen besiedeln, während für die Gemeine Fichte in den wärmeren Regionen Bayerns ein höheres Anbaurisiko bestehen könnte (Kölling et al. 2007). Wegen ihrer Einfachheit (Bolte et al. 2008) und aufgrund der eingeschränkten Modell- und Szenarienauswahl stellen die grafischen Klimahüllen von Kölling (2007) nur einen ersten Schritt auf dem Weg zu belastbaren Aussagen über die Zukunft der Artenzusammensetzung unserer Wälder dar.

Komplexere Klimahüllenmodelle berücksichtigen viele verschiedene Klimaparameter und berechnen, wie groß die Wahrscheinlichkeit ist, dass bestimmte Baumarten in einer Region vorkommen. Demnach liegt die Verbreitungsgrenze von Stielund Traubeneiche (Quercus robur und Quercus petraea) und 
Waldkiefern (Pinus sylvestris) im Zeitraum 2071-2100 (Szenario SRES A2) deutlich weiter im Osten und Norden als heute (Henschel 2008). Im westlichen Teil Deutschlands wachsen dann wesentlich weniger Kiefern.

Modelle zur Baumartenverteilung sind statistische Modelle auf der Basis von Inventurdaten der deutschen Bundeswaldinventur BWI II (Hanewinkel et al. 2010; Falk und Mellert 2011) oder der Monitoringdaten des ICP Forests (International Co-operative Programme on Assessment and Monitoring of Air Pollution Effects on Forests) (Hanewinkel et al. 2012; Meier et al. 2012). Sie zeigen, wie sich Anbaueignung und -risiko einzelner Baumarten regional verschieben. Berechnungen ergaben: Im Vergleich zum Jahr 2000 nehmen die geeigneten Flächen für Fichtenanbau in Baden-Württemberg bis 2030 um $21 \%$ ab (SRES-Szenario B1), bis 2100 um $93 \%$ (SRES-Szenario A2) (Hanewinkel et al. 2010). In Fläche umgerechnet entspricht dies einem Rückgang von 190.000 bzw. 860.000 ha. Wolfgang Falk und Karl Mellert von der Bayerischen Landesanstalt für Wald und Forstwirtschaft ermittelten für Bayern anhand zweier verschiedener Baumartenverteilungsmodelle für das Szenario B1 (Modell WETTREG), dass die Zahl der Flächen mit geringer Anbaueignung - also hohem Anbaurisiko - der Weißtanne (Abies alba) zunimmt. Nur auf einigen Gebirgsstandorten, z. B. in den Bayerischen Alpen oder im Bayerischen Wald, kann in Zukunft die Anbaueignung der Weißtanne zunehmen (Falk und Mellert 2011).

Mit einem Vegetationsmodell wurde die Dynamik der häufigsten europäischen Baumarten bei Klimaveränderungen untersucht (Hickler et al. 2012). Simulationen bis 2085 (A2-SRES-Szenario mit zwei globalen Zirkulationsmodellen) zufolge wandelt sich die Vegetation in weiten Teilen Europas deutlich - das wirkt sich auf die Bewirtschaftung von Wäldern und auf Schutzgebiete aus. Demnach weisen 31-42\% von Europa im Jahr 2085 einen anderen Vegetationstyp auf. Als ein Hotspot der Veränderung wurde die Übergangszone zwischen den Laubwäldern der gemäBigten Breiten und borealen Nadelwäldern in Nordeuropa und den höheren Berglagen Zentraleuropas ausgemacht. Diese Untersuchungen berücksichtigen allerdings nicht eine natürliche lokale Anpassung der einzelnen Arten, die dazu führen kann, dass die Veränderung der Vegetationstypen weniger drastisch ausfällt.

Mit kombinierten Modellen für Baumartenverteilung und Wald lassen sich die erforderlichen Migrationsraten für die Areale verschiedener Baumarten in Europa schätzen (Meier et al. 2012). Das Ergebnis: Außer der Waldkiefer können alle in den Alpen vorkommende Arten neu entstandene Areale erschließen. Dagegen bleibt die Baumverteilung in Nord- und Südeuropa recht stabil. Bei den Pionierbaumarten, die auf kargen und sonnenreichen Standorten die Erstbesiedlung vornehmen, liegen die Migrationsraten sehr hoch, bei den Klimaxbaumarten, die das Endstadium der natürlichen Sukzession im Wald darstellen, nur etwa ein Zehntel so hoch. Während des 21. Jahrhunderts gehen die mittleren Migrationsraten überall zurück, allerdings bei den Pionierbaumarten geringer als bei den Klimaxbaumarten.

In Sachsen und Thüringen haben die Forstverwaltungen verschiedene Zusammensetzungen von Baubeständen unter Berücksichtigung unterschiedlicher Emissionsszenarien mithilfe des Habitatmodells BERN simuliert. Dabei setzten sie Standorteigenschaften wie Boden und Klima in Beziehung zu aktuell vorkommenden Waldgesellschaften und berechneten ihre künftige Vorkommenswahrscheinlichkeit mittels beispielhafter Projektionen des Regionalmodells WETTREG für Sachsen (B1SRES-Szenario) bzw. Thüringen (A1B-Szenario). Besonders in den Mittelgebirgen gibt es demnach künftig weniger Potenzial für Fichtenwälder. Stattdessen finden dort Buchen gute Wuchsbedingungen. In den Tieflagen lösen Eichenwälder die Buchenwälder ab. Daher plant man, in Sachsen und Thüringen in den Bergregionen mehr Buchen, im Tiefland mehr Eichen anzubauen. Da solche Planungen im forstlichen Bereich durch die langen Umtriebszeiten langfristige Konsequenzen haben, ist eine fortlaufende, kritische Überprüfung der gewonnen Erkenntnisse auf der Grundlage neuer Ergebnisse der Klimaforschung unabdingbar (Gemballa und Schlutow 2007; Eisenhauer und Sonnemann 2009; Schlutow et al. 2009; Frischbier et al. 2010). In Mitteleuropa wird der Eichenwald möglicherweise generell zunehmen, während Kiefern- und Fichtenwälder sich zurückziehen werden (Hanewinkel et al. 2012).

\subsubsection{Längere Vegetationsperioden}

In den vergangenen Jahrzehnten haben sich die Vegetationsperioden ausgedehnt (Menzel und Fabian 1999; Chmielewski und Rötzer 2001; Bissolli et al. 2005). So beginnen Laubaustrieb oder Blüte früher im Jahr, Laubverfärbung und Blattfall setzen dagegen später ein. Längere Vegetationsperioden können die Produktion von mehr Biomasse ermöglichen, wenn Nährstoffe ausreichend zur Verfügung stehen. Negative Auswirkungen sind aber ebenfalls möglich. Beispielsweise können die Wechselwirkungen zwischen Arten, etwa bei der Bestäubung, gestört werden (Menzel et al. 2006). Mildere Winter können die Frosthärte von Bäumen verringern und damit mehr Spätfrostschäden verursachen. Durch hohe Wintertemperaturen kann der Stoffwechsel während der Winterruhe aktiviert werden, was die Bäume schwächt (Kätzel 2008). Eine früher beginnende und später endende Wachstumsperiode kann nur dann in vermehrtes Wachstum umgesetzt werden, wenn die Bäume im Sommer nicht in Trockenstress geraten, weil das Bodenwasser früher verbraucht ist (Richardson et al. 2010). Längere Vegetationszeiten können sich auch auf durch bestimmte Wärmesummen limitierte Schadorganismen von Bäumen auswirken.

\subsubsection{Waldschäden: keine einfachen Antworten}

Der Klimawandel schädigt den Wald direkt oder indirekt (Möller 2009; Müller 2009; Petercord et al. 2009). Windbruch und Trockenstress beispielsweise sind direkte Schäden, ebenso vermehrter Schädlingsbefall durch verändertes Klima. Indirekt wirkt der Klimawandel, indem er die Empfänglichkeit (Prädisposition) der Bäume für schädliche Einflüsse verändert und sie z. B. stärker auf Schadfaktoren reagieren (Müller 2008, 2009). Zuverlässige Aussagen zu den direkten und indirekten Folgen des Klimawandels sind schwierig, weil es komplexe Wechselwirkungen zwischen potenziellen Wirtsbaumarten und dem Klimawandel gibt. Da- 
rüber hinaus lässt sich schwer beurteilen, wie potenzielle Schädlinge auf den Klimawandel reagieren. Sie haben zudem natürliche „Gegenspieler“, deren Reaktionen auf ein verändertes Klima ebenfalls zu berücksichtigen ist.

Die Unsicherheit von Vorhersagen ergibt sich außerdem aus den möglichen Arealverschiebungen der Baumarten. Schäden sind vor allem dann zu erwarten, wenn Baumarten infolge des Klimawandels in nun ungeeigneten Regionen verbleiben. Mit der Anpassung der Waldbewirtschaftung und durch natürliche Arealverschiebungen werden Baumarten aber auch in Regionen vorkommen, die für sie geeignet sind.

\section{- Mehr Kohlendioxid in der Atmosphäre}

Nach verschiedenen Klimaszenarien entwickelt sich die Kohlendioxidkonzentration in der Atmosphäre bis 2100 auf Werte zwischen 400 ppm - RCP8.5 - 1370 ppm (Vuuren et al. 2011). Direkte Baumschäden und direkte Wirkungen auf abiotische und biotische Schadfaktoren dadurch sind sehr unwahrscheinlich. Eher wirken höhere $\mathrm{CO}_{2}$-Konzentrationen indirekt, indem sie die Modifikation der Nahrungsqualität der Pflanzen steigern (Docherty et al. 1997; Veteli et al. 2002; Whittaker 1999). Tendenziell steigen Biomasseproduktion und Stoffumsatz der Pflanzen - eventuell wirkt sich das auf Risikostreuung und Verteidigung aus. Auch der Knospenaustrieb verändert sich, potenzielle Schadfaktoren treffen auf andere Entwicklungsphasen der Bäume.

\section{- Steigende Temperatur}

Die Erde wird wärmer: Die globale Oberflächentemperatur könnte bis $2100 \mathrm{um}$ bis zu $4,8^{\circ} \mathrm{C}$ steigen. Berücksichtigt man Unsicherheiten in den Klimaprojektionen, ist eine Steigerung um bis zu 7,8 ${ }^{\circ} \mathrm{C}$ möglich (IPCC 2014). Prinzipiell kann das direkt die Entwicklung von Insekten beeinflussen. Wärmere Blattoberflächen steigern die Fraßaktivität blattfressender Insekten, weniger Stickstoff in den Blättern dämpft oder stimuliert potenzielle Schadinsekten.

Nach der sogenannten Temperatursummenregel ist das Produkt aus wirksamer Temperatur und Entwicklungsdauer konstant (Schäfer 2003): je höher die Temperatur, desto schneller durchlaufen Insekten ihre Entwicklungsstadien. Unklar ist aber zumeist, ob sich das für eine bestimmte Art grundsätzlich positiv oder negativ auswirkt.

Bei stabilen Generationsfolgen von Insekten werden höhere Temperaturen vorrangig Entwicklungsstadien verkürzen, oder sie beginnen früher. Einige Insekten sind dadurch erfolgreicher und verursachen in kürzeren Zeitabständen Schäden. Hingegen verläuft etwa die Entwicklung der Forleule (Panolis flammea) ganz anders: Höhere Temperaturen in der Schwärmzeit verkürzen das Endstadium der Metamorphose, das Imaginalstadium. Dadurch kommt es zu einer unvollständigen Eiablage, und es gibt weniger Nachkommen (Escherich 1931; Majunke et al. 2000).

Bei variablen und temperaturgesteuerten Generationsfolgen ist bei höheren Temperaturen mit kürzeren Entwicklungszeiten oder einer Erhöhungen der Generationen pro Jahr zu rechnen. Schädlinge wie der Große braune Rüsselkäfer (Hylobius abietis) und der Blaue Kiefernprachtkäfer (Phaenops cyanea) dürften dann öfter als einjährige statt zweijährige Generationen vorkommen. Das bedeutendste mitteleuropäische rindenzerstörende (cambi- ophage) Insekt, der Große achtzähnige Fichtenborkenkäfer (Ips typographus), könnte zunehmend drei statt bisher zwei Generationen im Jahr hervorbringen. Doch wie stark würden diese Entwicklungen den Wald gefährden? Das ist selbst in diesen bekannten Fällen noch nicht abschließend untersucht (Schopf 1989). Die Schäden der Rüssel- und Kiefernprachtkäfer würde man zwar eventuell schnell sehen, aber es würden sich auch die Schadzeiträume verkürzen. Eine dritte Generation bei Ips typographus kann zusätzlichen Schaden anrichten. Das Ende der Eiablage im Spätsommer ist jedoch fotoperiodisch gesteuert (Schopf 1989). Nach bisherigen Erkenntnissen ist dieser Borkenkäfer nicht in der Lage, im Ei-, Larven- oder Puppenstadium zu überwintern (SchmidtVogt 1989), sodass sich eine nicht vollendete dritte Generation auch negativ auf die Populationsentwicklung auswirken könnte.

\section{- Invasive und partizipierende Arten}

Steigt die Temperatur in Mitteleuropa, könnten sich bislang unauffällige Arten besser entwickeln (partizipierende Arten), an wärmeres Klima angepasste Arten würden einwandern (invasive Arten). Zu diesen Gruppen gehören beispielsweise die Prozessionsspinner (Thaumetopoea sp.), die Prachtkäfer (Buprestidae) oder auch der Kieferholznematode (Bursaphelenchus xylophilus), der Schwarze Nutzholzborkenkäfer (Xyloborus germanus) (Immler und Blaschke 2007) sowie der Erreger des Kieferntriebsterbens (Spaeropsis sapinea) (Hänisch et al. 2006; Heydeck 2007). Dabei ist jedoch zu prüfen, ob Vorkommen und Anpassung dieser Arten tatsächlich mit dem Klimawandel zu tun haben.

\section{- Extreme Witterung}

Dürren, Überflutungen und Stürme können direkt den Wald schädigen. Sturm ist der bedeutendste direkte Schadfaktor in deutschen Wäldern. In den vergangenen Jahren nahmen Sturmschäden deutlich zu (Majunke et al. 2008). In deutschen Wäldern steigt zudem der Anteil sturmgefährdeter mittelalter und alter Bestände - sogar ohne Zunahme von Witterungsextremen muss man deshalb mit mehr Schadholz rechnen.

Durch Witterungsextreme - vor allem Niederschlag und Stürme - entsteht darüber hinaus jedoch auch eine höhere Prädispositionen der Bäume für schädigende Insekten. Mit den heutigen Aufarbeitungstechnologien lassen sich Massenvermehrungen von Borkenkäfern nach Dürre und Stürmen jedoch immer besser verhindern.

\section{- Arealverschiebungen und Waldbrandgefahr}

Aus den klimabedingten Arealverschiebungen von Bäumen ergeben sich Gebiete, in denen die entsprechenden Baumarten künftig nicht mehr existieren können (Krisengebiete). Zudem entstehen Gebiete, in denen sich bestimmte Arten neu ansiedeln werden (Initialgebiete). In den Krisengebieten werden Bäume empfindlicher gegenüber biotischen Schadfaktoren und von diesen vermehrt heimgesucht. Außerdem werden sich Folgeorganismen, die bisher kaum in Erscheinung traten, stärker zu Erstbesiedlern entwickeln. In Initialgebieten, in denen sich die andernorts verdrängten Baumarten neu ansiedeln oder angebaut werden, bilden sich neue Lebensräume. Dorthin expandieren auch potenzielle Schadorganismen, deren Population nach einer Anfangsphase exponentiell wächst und sich an den neuen 
Mehr Kohlenstoff speichern - aber wie? Unter rein ökologischen Gesichtspunkten wäre eine Nichtnutzung der Wälder mit zunächst einhergehendem Aufbau des Kohlenstoffspeichers eine mögliche Option. Jedoch ist die Speicherfähigkeit auf einen Gleichgewichtszustand begrenzt (Kohlenstoffabnahme durch Mortalität und Zersetzung entspricht $C$ Sequestrierung) und schließt Substitutionseffekte aus. Unter den realen gesellschaftlichen Anforderungen an den Wald könnte vor allem durch neue Bewirtschaftungsstrategien und Kaskadennutzung von Holzprodukten mehr Kohlenstoff gespeichert werden als durch eine Nichtnutzung. Dabei spielen verschiedene Instrumente zur Intensivierung der Holzproduktion eine wichtige Rolle:
- Ernte der Bäume zum Zeitpunkt des maximalen Gesamtzuwachses, inklusive Mehrfachnutzung des Holzes und Ersatz von energieintensiveren Materialien durch Holz und energetischer Endnutzung zur Substitution von fossilen Brennstoffen.

- Frühzeitige Verjüngung von überalterten Beständen bzw. Beständen mit überdurchschnittlich großer Vorratshaltung.

- Baumartenwechsel hin zu leistungsstarken und raschwüchsigen Arten.

- Überführung von Altersklassenwäldern in Dauerwälder.

Auch bei den alternativen Bewirtschaftungen und anschließender Kaskadennutzung der Holzprodukte sind der Senkenleistung von Wäldern natürliche Grenzen gesetzt. So müsste eine klimafreundliche und klimaangepasste Bewirtschaftung von Beständen im Einzelfall geplant und umgesetzt werden, da die lokalen Standortverhältnisse berücksichtigt werden müssen (Krug et al. 2010). Durch Ausgleichs- oder Ersatzmaßnahmen in Form von Aufforstung, z. B. von landwirtschaftlichen Grenzertragsflächen, kann allerdings zusätzliche Speicher- und Senkenleistung geschaffen werden. Jedoch bestehen hier vielfältige konkurrierende Nutzungsinteressen und hohe rechtliche und gesellschaftliche Hindernisse.
Lebensraum anpasst. Ein markantes Beispiel: Verschiedene Arten von Kurzschwanzmäusen besiedeln bereits heute Waldumbauflächen.

Die von Klimaszenarien skizzierten Änderungen, u. a. Wassermangel, erhöhen die Waldbrandgefahr (Badeck et al. 2004; Hänisch et al. 2006; SMUL 2008). Aber die Waldstrukturen verändern sich, sodass die besonders gefährlichen und schwer bekämpfbaren Vollfeuer - das sind Brände vom Boden bis zur Kronenschicht unwahrscheinlicher werden könnten. Außerdem sind Waldbrandvorbeugung, -überwachung und -bekämpfung sehr effektiv. Wird der Schutz vor Waldbränden beibehalten und weiterentwickelt, lässt sich die Waldbrandgefahr trotz Klimawandels in Deutschland wahrscheinlich beherrschen (Müller 2004, 2008, 2009).

\subsubsection{Temperatur und Niederschläge beeinflussen Produktivität}

Die Hitzewelle im Sommer 2003 lässt die Auswirkungen des Klimawandels auf den Wald erahnen. Der Kronenzustand als ein Indikator für die Vitalität eines Baumes hat sich 2003 bei den meisten Baumarten deutlich verschlechtert - Trockenheit und Hitze mit den damit verbundenen Wassermangelerscheinungen sind dafür eine plausible Erklärung (ICP 2004). Eine Projektion mit mehreren globalen Klimamodellen weist jedoch darauf hin, dass durch den zu erwartenden Klimawandel europaweit etwas mehr Flächen entstehen, auf denen Wald gedeihen kann (Schröter et al. 2005). Neben dem Management (Köhl et al. 2010) beeinflusst wohl auch die Änderung der Niederschläge entscheidend die Produktivität von Wald. Nehmen die Niederschläge zu, könnte in Deutschland bei drei von vier Hauptbaumarten die Produktivität um bis zu $7 \%$ steigen. Wird es eher trockener, geht die Produktivität besonders an wasserarmen Standorten um 4-16\% zurück (Lasch et al. 2005; Lindner et al. 2010). Der Sommer 2003 hat zudem gezeigt: Bei Trockenheit und Wassermangel betreiben Wälder weniger Fotosynthese und mehr Atmung, sodass sie zu $\mathrm{CO}_{2}$-Quellen werden (Dobbertin und de Vries 2008; Kap. 17). Damit wird in den Trockenperioden die Kohlenstoffspeicherleistung der Wälder vermindert.

\subsubsection{Kohlenstoffhaushalt: von der Senke zur Quelle}

Seit 1990 nimmt die Leistung des Waldes als Kohlenstoffsenke auf bewirtschafteten Flächen in Deutschland ab (Krug et al. 2009). Diese Entwicklung ist einerseits dem Altersklassenaufbau der Aufforstungen nach dem 2. Weltkrieg geschuldet - damals wurden durch Insektenbefall und die sog. "Reparationshiebe“ der Alliierten zerstörte Wälder wieder aufgeforstet. Andererseits resultiert die verringerte Senkenleistung aus der zyklischen Nutzung. Spätestens seit 2002 werden der Vorratsaufbau und vergangene Mindernutzungen verstärkt mobilisiert und führen zu einer stetigen Abnahme der Senkenleistung, da kurz- bis mittelfristig mehr Kohlenstoff durch Altbestandsernte freigesetzt wird, als durch nachwachsende Jungbestände sequestriert werden kann.

Auf Basis der Waldentwicklungs- und Holzaufkommensmodellierung (WEHAM) wurde unabhängig von den Auswirkungen des Klimawandels projiziert, dass der deutsche Wald in den kommenden vier Jahrzehnten von einer $\mathrm{CO}_{2}$-Senke zu einer $\mathrm{CO}_{2}$-Quelle werden wird (Dunger et al. 2005; Dunger und Rock 2009; Polley und Kroiher 2006; Krug et al. 2010).

\subsection{Anpassung in der Forstwirtschaft}

Die Anpassung der Bewirtschaftung an den Klimawandel zielt auf eine höhere Vitalität von Wäldern. Anpassungen an Niederschlagsdefizite und höhere Temperaturen lassen sich mit Veränderungen in der Baumartenwahl (Lasch et al. 2005), der Bestandsstruktur etwa durch geringere Stammzahlen, neuen Durchforstungsmethoden, Verjüngungskonzepten oder im Anbau trockenresistenter Herkünfte realisieren (Reyer et al. 2009).

In der Forstwirtschaft sind Entscheidungen langfristig. Zudem bestehen komplexe Wechselwirkungen zwischen dem regionalen Klimawandel und ökologischen, ökonomischen sowie sozialen Faktoren. Das alles erhöht das Produktionsrisiko im Wald (Taeger et al. 2013). Somit hängt viel von der Auswahl 


\section{Gewinnmaximierer}

\section{Waldreinertragsmaximierer}

\section{Zielstärkennutzer}
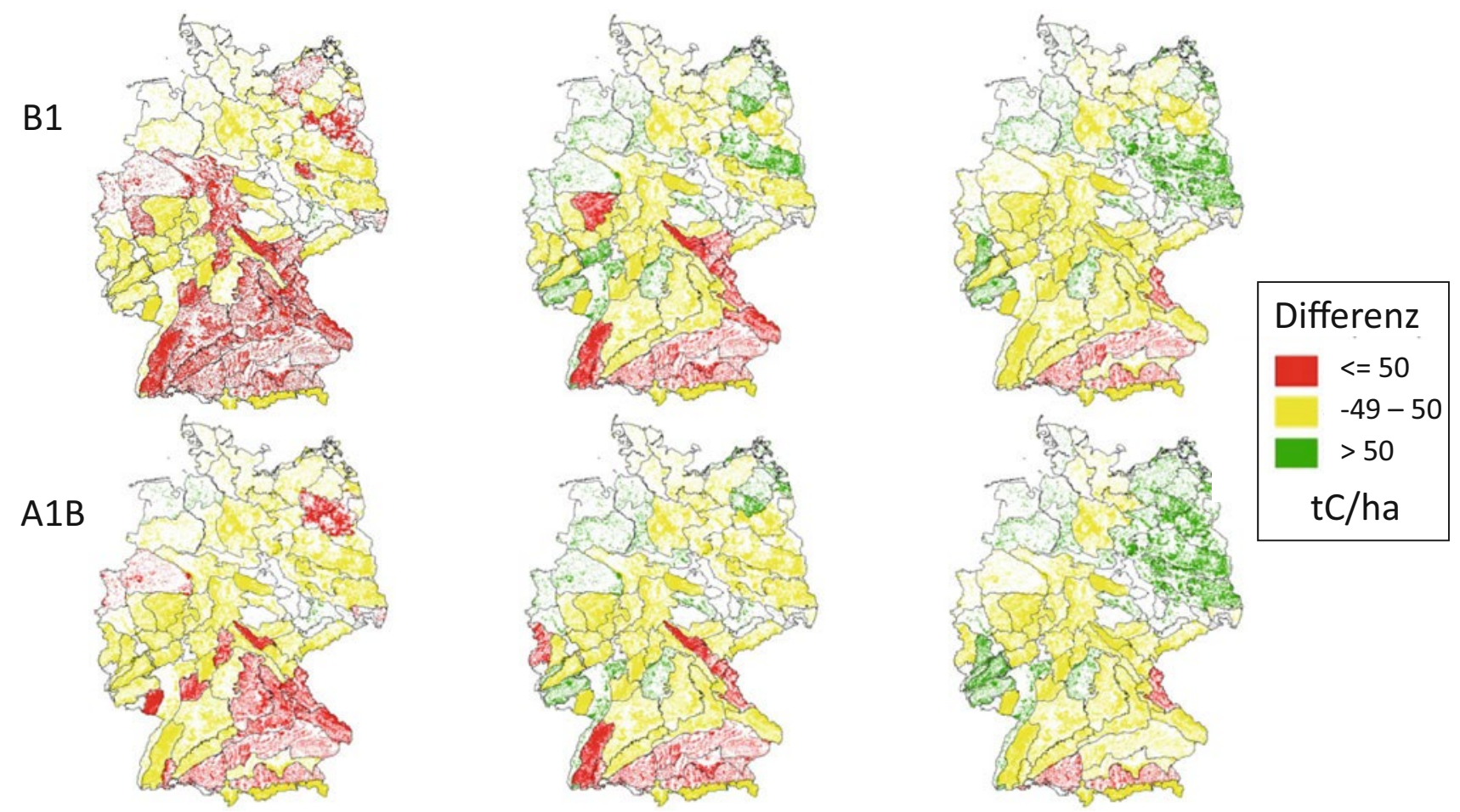

D Abb. 19.2 Veränderung des Waldkohlenstoffspeichers [tC/ha] zwischen 2000 und 2100 nach Waldbewirtschaftungstypen und Klimaszenarien (rot Speicherabnahme, grün Speicherzunahme, gelb keine Speicheränderung)

der richtigen Baumarten oder den richtigen Herkünften bereits angebauter Arten ab, die mit den erwarteten Umweltbedingungen besser zurechtkommen (Bolte et al. 2009). Mit dem Anbau trockenstressresistenter Pflanzen lässt sich die Forstwirtschaft offenbar an den Klimawandel anpassen (Fyllas und Troumbis 2009; Temperli et al. 2012). Jedoch müssen Auswahl und Mischung der Baumarten regional betrachtet werden. Standortbedingungen, der projizierte regionale Klimawandel und die Reaktion der einzelnen Arten darauf müssen berücksichtigt werden (Temperli et al. 2012). So wurde im Zuge großflächiger Aufforstungen nach den „Reparationshieben“ ( Abschn. 19.2.5) die trockenanfällige Gemeine Fichte in klimatisch nur bedingt geeigneten Gebieten angebaut, sodass es heute ein Ungleichgewicht zwischen Standort und Klimabedingungen gibt. Durch den Klimawandel wird sich dieses verstärken und auf weitere Gebiete ausbreiten (Ludemann 2010).

Köhl et al. (2010) haben die Auswirkungen von unterschiedlichen Bewirtschaftungszielen bei verschiedenen Klimaszenarien untersucht. Die drei Waldbewirtschaftungstypen haben unterschiedliche Ziele: Der "Gewinnmaximierer" nutzt den Wald, sobald der Wertzuwachs unter $2 \%$ sinkt. Der „Waldreinertragsmaximierer“ nutzt den Wald beim Maximum des mittleren jährlichen Ertrags. Und der „Zielstärkennutzer“ bewegt sich nah an naturnaher Waldwirtschaft und nutzt Bäume ab einem definierten Zieldurchmesser. Das Ergebnis: Je nach Bewirtschaftungstyp und Klimaszenario verändert sich von 2000 bis 2100 neben der Holzproduktion und der Baumartenzusammensetzung auch die Menge an Waldkohlenstoff (- Abb. 19.2). Dabei beeinflusst unter den in der Studie vorausgesetzten Randbedingungen die Waldbewirtschaftung die künftige Bestandsentwicklung stärker als der Klimawandel. Ähnliches wurde für boreale Wälder berichtet (Alam et al. 2008; Garcia-Gonzalo et al. 2007; Briceño-Elizondo et al. 2006).

Da Anpassungsplanungen im forstlichen Bereich langfristige Konsequenzen haben, ist eine fortlaufende, kritische Überprüfung der gewonnenen Erkenntnisse auf der Grundlage neuer Ergebnisse der Klimaforschung mit mehreren und neueren Modell- und Szenarienensembles unabdingbar (Krug et al. 2010a).

\subsection{Kurz gesagt}

In der Vergangenheit haben sich Wälder an die geringen Veränderungen des am Wuchsort herrschenden Klimas angepasst. Die gegenwärtige Geschwindigkeit des Klimawandels in Verbindung mit der aktuellen Verteilung der Baumarten überfordert jedoch die natürliche Anpassung. Vegetationszonen, Verbreitungsgebiete der Baumarten und Artzusammensetzung der Wälder verschieben sich. Sowohl die höheren Temperaturen als auch die veränderte Verteilung der Niederschläge sowie zunehmende Extremereignisse werden sich auf die Waldökosysteme auswirken. Trockenstress durch weniger Sommerniederschläge und die dadurch beschleunigte Entwicklung von Insekten sowie die steigende Gefahr von Waldbränden und Stürmen werden die Anfälligkeit der Bäume erhöhen. 
In Mitteleuropa wird der Eichenwald zunehmen, beginnend in den Tieflagen. Der Buchenwald wandert von den Tieflagen in die Mittelgebirge. Dort werden sich die Kiefern- und Fichtenwälder allmählich zurückziehen.

Bei Anpassungsstrategien spielen somit die Waldbewirtschaftung sowie die standort- und klimaangepasste Auswahl der Baumarten eine große Rolle. Geeignete Strategien berücksichtigen auch die Produktion des Rohstoffs und Energieträgers Holz. So lässt sich die Funktion von Wäldern als Zwischenspeicher im globalen Kohlenstoffkreislauf sichern und fördern. Holzprodukte können zudem energieintensive Materialien und fossile Energieträger ersetzen und zu einer Reduktion der Treibhausgasemissionen beitragen.

\section{Literatur}

Alam A, Kilpeläinen A, Kellomäki S (2008) Impacts of thinning on growth, timber production and carbon stocks in Finland under changing climate. Scand J Forest Res 23:501-512

Badeck FW, Lasch P, Hauf Y, Rock J, Suckow F, Thonicke K (2004) Steigendes klimatisches Waldbrandrisiko. AFZ-Der Wald 59:90-93

Bergman R, Puettmann M, Taylor A, Skog KE (2014) The Carbon Impacts of Wood Products. For Prod J 64:220-231

Bissolli P, Müller-Westermeier G, Dittmann E, Remisová V, Braslavská O, Stastný P (2005) 50-year time series of phenological phases in Germany and Slovakia: a statistical comparison. Meteorol Z 14(2):173-182

Block J, Gauer J (2012) Waldbodenzustand in Rheinland-Pfalz: Ergebnisse der zweiten landesweiten Bodenzustandserhebung BZE II. Mitt Forschungsanst Waldökologie und Forstwirtschaft Rheinland-Pfalz 70:228

BMEL (2014) Der Wald in Deutschland. Ausgewählte Ergebnisse der dritten Bundeswaldinventur. Bundesministerium für Ernährung und Landwirtschaft, Berlin

BMELV (2009) Waldbericht der Bundesregierung 2009. Bundesministerium für Ernährung, Landwirtschaft und Verbraucherschutz, Berlin

Bolte A, Ibisch P, Menzel A, Rothe A (2008) Was Klimahüllen uns verschweigen. AFZ-Der Wald 15:800-803

Bolte A, Ammer C, Löf M, Madsen P, Nabuurs G-J, Schall P et al (2009) Adaptive forest management in central Europe: climate change impacts, strategies and integrative concept. Scand J Forest Res 24:473-482

Briceño-Elizondo E, Garcia-Gonzalo J, Peltola H, Kellomäki S (2006) Carbon stocks and timber yield in two boreal forest ecosystems under current and changing climatic conditions subjected to varying management regimes. Environ Sci Policy 9(3):237-252

Chmielewski FM, Rötzer T (2001) Response of tree ponology to climate change across Europe. Agric For Meteorol 108:101-112

Dobbertin M, de Vries W (2008) Interactions between climate change and forest ecosystems. In: Fischer R (Hrsg) Forest ecosystems in a changing environment: identifying future monitoring and research needs Report and Recommendations - COST Strategic Workshop, 11-13 March 2008. Stueber Grafik, Göttingen, S 8-12

Docherty M, Salt DT, Holopainen JK (1997) The impacts of climate change and pollution on forest pests. In: Watt AD, Stork NE, Hunter MD (Hrsg) Forests and Insects. Chapman \& Hall, London

Dunger K, Rock J (2009) Projektionen zum potentiellen Rohholzaufkommen. AFZ-Der Wald 64(20):1079-1081

Dunger K, Bösch B, Polley H (2005) Das potentielle Rohholzaufkommen 2002 bis 2022 in Deutschland. AFZ 60(3):114-116

Dunger K, Stümer W, Oehmichen K, Riedel T, Ziche D, Grüneberg E, Wellbrock N (2014) Nationaler Inventarbericht Deutschland. Kapitel 7.2 Wälder. Umweltbundesamt (UBA), Dessau-Roßlau

Eisenhauer D-R, Sonnemann S (2009) Waldbaustrategien unter sich ändernden Umweltbedingungen - Leitbilder, Zielsystem, Waldentwicklungstypen. Waldökol Landschaftsforsch Natursch 8:71-88

Escherich K (1931) Die Forstinsekten Mitteleuropas. Raul Parey, Berlin
Falk W, Mellert KH (2011) Species distribution models as a tool for forest management planning under climate change: risk evaluation of Abies alba in Bavaria. J Veg Sci 22(4):621-634

FAO (2010) Global Forest Resources Assessment 2010: Main Report, Rome. FAO Forestry Paper

Forest Europe (2011) State of Europe's Forests. UN-ECE, UN-FAO, FOREST EUROPE Liaison Unit Oslo. Oslo, Geneva, Rome:337

Frischbier N, Profft I, Arenhövel W (2010) Die Ausweisung klimawandelangepasster Bestandeszieltypen für Thüringen. Forst Holz 65(2):28-35

Fyllas N, Troumbis A (2009) Simulating vegetation shifts in north-eastern Mediterranean mountain forests under climatic change scenarios. Global Ecology and Biogeography 18(1):64-77

Garcia-Gonzalo J, Peltola H, Briceño-Elizondo E, Kellomäki S (2007) Effects of climate change and management on timber yield in boreal forests, with economic implications: a case study. Clim Chang 81:431-454

Gemballa R, Schlutow A (2007) Überarbeitung der Forstlichen Klimagliederung Sachsens. AFZ-Der Wald 15:822-826

Hanewinkel M, Hummel S, Cullmann DA (2010) Modelling and economic evaluation of forest biome shifts under climate change in Southwest Germany. For Ecol Manage 259(4):710-719

Hanewinkel M, Cullmann DA, Schelhaas MJ, Nabuurs GJ, Zimmermann NE (2012) Climate change may cause severe loss in the economic value of European forest land. Nat Clim Chang 3(3):203-207

Hänisch T, Kehr R, Schubert O (2006) Schwarzkiefer auf Muschelkalk trotz Sphaeropsis-Befall? AFZ-Der Wald 61:227-230

Henschel A (2008) Habitatmodellierung der drei Baumarten Waldkiefer, Traubeneiche und Stieleiche. Geographisches Institut. Berlin, Humboldt-Universität. Diplom, $119 \mathrm{~S}$

Heydeck P (2007) Pilzliche und pilzähnliche Organismen als Krankheitserreger an Kiefern. In: Ministerium für Ländliche Entwicklung, Umwelt und Verbraucherschutz des Landes Brandenburg (Hrsg) Die Kiefer im nordostdeutschen Tiefland - Ökologie und Bewirtschaftung. Eberswalder Forstliche Schriftenreihe, Bd. XXXII. Verlagsgesellschaft, Potsdam

Hickler T, Vohland K, Feehan J, Miller PA, Smith B, Costa L, Giesecke T, Fronzek S, Carter TR, Cramer W, Kuhn I, Sykes MT (2012) Projecting the future distribution of European potential natural vegetation zones with a generalized, tree species-based dynamic vegetation model. Glob Ecol Biogeogr 21(1):50-63

Houghton RA (2013) The emissions of carbon from deforestation and degradation in the tropics: past trends and future potential. Carbon Manag 4:539-546

ICP (2004) The condition of forests in Europe: 2004 Executive Report, Geneva Immler T, Blaschke M (2007) Forstschädlinge profitieren vom Klimawandel. LWF aktuell 14(5):24-26

IPCC (2007) Intergovernmental Panel on Climate Change: Climate Change 2007 - The physical science basis. Contribution of Working Group 1 to the Fourth Assessment Report of the IPCC

IPCC (2014) Climate change 2014: Synthesis report. Contribution of Working groups I, II and III to the Fifth Assessment Report of the Intergovernmental Panel on Climate Change, Geneva

Kätzel R (2008) Klimawandel. Zur genetischen und physiologischen Anpassungsfähigkeit der Baumarten. Arch Forstwes Landschaftsökol 42:9-15

Knauf M, Köhl M, Mues V, Olschofsky K, Frühwald A (2015) Modeling the $\mathrm{CO}_{2}{ }^{-}$ effects of forest management and wood usage on a regionl basis: Carbon Balance and Management 10:13

Köhl M, Hildebrandt R, Olschofksy K, Köhler R, Rötzer T, Mette T, Pretzsch H, Köthke M, Dieter M, Mengistu A, Makeschin F, Kenter B (2010) Combating the effects of climatic change on forests by mitigation strategies. Carbon Balance Manag 5(8)

Kölling C (2007) Klimahüllen für 27 Waldbaumarten. AFZ-Der Wald 23:12421245

Kölling C, Zimmermann L, Walentowski H (2007) Klimawandel: Was geschieht mit Buche und Fichte? AFZ-Der Wald 11:584-588

Krug J et al (2009) Options for accounting carbon sequestration in German forests. Carbon Balance Manag 4:5

Krug J, Köhl M (2010a) Bedeutung der deutschen Forstwirtschaft in der Klimapolitik. AFZ-Der Wald 65(17):30-33

Krug J, Kriebitzsch W-U, Riedel T, Olschofsky K, Bolte A, Polley H, Stümer W, Rock J, Öhmichen K, Kroiher F, Wellbrock N (2010b) Potenziale zur Ver- 
meidung von Emissionen sowie der zusätzlichen Sequestrierung im Wald und daraus resultierenden Fördermaßnahmen. Studie im Auftrag des Bundesministeriums für Ernährung, Landwirtschaft und Verbraucherschutz. Thünen-Institut, Hamburg

Kurz WA, Dymond CC, Stinson G, Rampley GJ, Neilson ET, Carroll AL, Ebata T, Safranyik $L$ (2008) Mountain pine beetle and forest carbon feedback to climate change. Nature 452:987-990

Lal R (2005) Forest soils and carbon sequestration. For Ecol Manage 220:242258

Lasch P, Badeck F, Suckow F, Lindner M, Mohr P (2005) Model-based analysis of management alternatives at stand and regional level in Brandenburg (Germany). For Ecol Manage 207(1-2):59-74

Lindner M, Maroschek M, Netherer S, Kremer A, Barbati A, Garcia-Gonzalo J, Seidl R, Delzon S, Corona P, Kolstrom M, Lexer MJ, Marchetti M (2010) Climate change impacts, adaptive capacity, and vulnerability of European forest ecosystems. For Ecol Manage 259(4):698-709

Ludemann T (2010) Past fuel wood exploitation and natural forest vegetation in the Black Forest, the Vosges and neighbouring regions in western Central Europe. Palaeogeogr Palaeoclimatol Palaeoecol 291:154-165

Luyssaert S, Schulze E-D, Börner A, Knohl A, Hessenmöller D, Law BE, Ciais P, Grace J (2008) Old-growth forests as global carbon sinks. Nature 455:213215

Majunke C, Möller K, Funke M (2000) Zur Massenvermehrung der Forleule (Panolis flammea Schiff., Lepidoptera, Noctuidae) in Brandenburg. Forstwirtsch Landsch ökol 34:127-132

Majunke C, Matz S, Müller M (2008) Sturmschäden in Deutschlands Wäldern von 1920 bis 2007. AFZ-Der Wald 63:380-381

Meier ES, Lischke H, Schmatz DR, Zimmermann NE (2012) Climate, competition and connectivity affect future migration and ranges of European trees. Glob Ecol Biogeogr 21(2):164-178

Menzel A, Fabian P (1999) Growing season extended in Europe. Nature 452:987990 (397:6721)

Menzel A, Sparks TH, Estrella N, Roy DB (2006) Altered geographical and temporal variability in response to climate change. Glob Ecol Biogeogr 15:498-504

Mitchell SR, Harmon ME, O'Connell, Kari EB (2012) Carbon debt and carbon sequestration parity in forest bioenergy production. GCB Bioenergy 4:818-827

Möller K (2009) Aktuelle Waldschutzprobleme und Risikomanagement in Brandenburgs Wäldern. Eberswalder Forstliche Schriftenreihe 42:63-72

Müller M (2004) Klimawandel - Auswirkungen auf abiotische Schadeinflüsse und auf Waldbrände sowie mögliche forstliche Anpassungsstrategien. In: Brandenburgischer Forstverein e. V. (Hrsg) Klimawandel - Wie soll der Wald der Zukunft aussehen?. Brandenburgischer Forstverein, Eberswalde, S 45-55

Müller M (2008) Grundsätzliche Überlegungen zu den Auswirkungen eines Klimawandels auf potenzielle Schadfaktoren in mitteleuropäischen Wäldern. In: Forstverein Mecklenburg-Vorpommern e. V. (Hrsg) Tagungsberichte, S 152-161

Müller M (2009) Auswirkungen des Klimawandels auf ausgewählte Schadfaktoren in den deutschen Wäldern. Wiss Zeitschr TU Dresden 58(3-4):69-75

Oehmichen K, Demant B, Dunger K, Grüneberg E, Hennig P, Kroiher F, Neubauer M, Polley H, Riedel T, Rock J, Schwitzgebel F, Stümer W, Wellbrock N, Ziche D, Bolte A (2011) Inventurstudie 2008 und Treibhausgasinventar Wald. Landbauforschung vTI agriculture and forestry research. Sonderheft, Bd. 343. Thünen-Institut, Braunschweig

Pan Y, Birdsey RA, Fang J, Houghton R, Kauppi PE, Kurz WA et al (2011) A large and persistent carbon sink in the world's forests. Science 333:988-993

Petercord R, Leonhard S, Muck M, Lemme H, Lobinger G, Immler T, Konnert M (2009) Klimaänderung und Forstschädlinge. LWF aktuell 72:4-7

Polley H, Kroiher F (2006) Struktur und regionale Verteilung des Holzvorrates und des potenziellen Rohholzaufkommens in Deutschland im Rahmen der Clusterstudie Forst- und Holzwirtschaft. Inst Waldökol Waldinvent. BFH, Eberswalde, S 128

Reyer C, Guericke M, Ibisch PL (2009) Climate change mitigation via afforestation, reforestation and deforestation avoidance - and what about adaptation to environmental change? New For 38:15-34

Richardson et al (2010) Influence of spring and autumn phenological transitions on forest ecosystem productivity. Phil Trans R Soc 365:3227-3246
Russ A, Riek W (2011) Pedotransferfunktionen zur Ableitung der nutzbaren Feldkapazität - Validierung für Waldböden des nordostdeutschen Tieflands. Waldökologie, Landschaftsforschung und Naturschutz 11:5-17

Schäfer M (2003) Wörterbuch der Ökologie. Spektrum Akademischer Verlag, Heidelberg und Berlin

Schlutow A, Profft I, Frischbier N (2009) Das BERN-Modell als Instrument zur Einschätzung der Angepasstheit von Waldgesellschaften und Baumarten an den Klimawandel in Thüringen. Forst Holz 64(4):31-33

Schmidt-Vogt H (1989) Krankheiten, Schäden, Fichtensterben. Die Fichte, Bd. 2. (Teil 2)

Schopf A (1989) Die Wirkung der Photoperiode auf die Induktion der Imaginaldiapause von Ips typographus (L) (Col, Scolytidae). J Appl Entomol 107:275-288

Schreck M, Lackner C, Walli AM (2016) Österreichs Wald. Bundesforschungsund Ausbildungszentrum für Wald, Naturgefahren und Landschaft, Wien, (85):28

Schroeter D, Cramer W, Leemans R, Prentice I, Araújo M, Arnell A et al (2005) Ecosystem Service Supply and Vulnerability to Global Change in Europe. Science 310:1333-1337

Settele J, Scholes R, Betts R, Bunn SE, Leadley D, Nepstad D et al (2014) Terrestrial and inland water systems. In: IPCC (Hrsg) Climate Change 2014. Impacts, adaptation and vulnerability. Part A: Global and sectoral aspects. Contribution of Working Group II to the Fifth Assessment Report of the Intergovernmental Panel on Climate Change. Cambridge University Press, Cambridge, S 271-359

SMUL - Sächsisches Staatsministerium für Umwelt und Landwirtschaft (2008) Sachsen im Klimawandel. Thieme und Co KG, Meißen

Taeger S, Zang C, Liesebach M, Schneck V, Menzel A (2013) Impact of climate and drought events on the growth of Scots pine (Pinus sylvestris L.) provenances. For Ecol Manage 307:30-42

Temperli C, Bugmann H, Elkin C (2012) Adaptive management for competing forest goods and services under climate change. Ecol Appl 22:2065-2077

UBA (2011) Berichterstattung unter der Klimarahmenkonvention der Vereinten Nationen und dem Kyoto-Protokoll. Nationaler Inventarbericht zum Deutschen Treibhausgasinventar. Climate Change 11:1990-2009

Veteli TO, Kuokkanen K, Julkunen-Tiitto R, Roininen H, Tahvanainen J (2002) Effects of elevated $\mathrm{CO}_{2}$ and temperature on plant growth and herbivore defensive chemistry. Glob Chang Biol 8:1240-1252

Vuuren DP van, Edmonds J, Kainuma M, Riahi K, Thomson A, Hibbard K et al (2011) The representative concentration pathways: an overview. Clim Chang 109:5-31

Whittaker JB (1999) Impacts and responses at population level of herbivorous insects to elevated $\mathrm{CO}_{2}$. Eur J Entomol 96:149-156

Open Access Dieses Kapitel wird unter der Creative Commons Namensnennung 4.0 International Lizenz (http://creativecommons.org/ licenses/by/4.0/deed.de) veröffentlicht, welche die Nutzung, Vervielfältigung, Bearbeitung, Verbreitung und Wiedergabe in jeglichem Medium und Format erlaubt, sofern Sie den/die ursprünglichen Autor(en) und die Quelle ordnungsgemäß nennen, einen Link zur Creative Commons Lizenz beifügen und angeben, ob Änderungen vorgenommen wurden.

Etwaige Abbildungen oder sonstiges Drittmaterial unterliegen ebenfalls der genannten Creative Commons Lizenz, sofern sich aus der Abbildungslegende oder der Quellreferenz nichts anderes ergibt. Sofern solches Drittmaterial nicht unter der genannten Creative Commons Lizenz steht, ist eine Vervielfältigung, Bearbeitung oder öffentliche Wiedergabe nur mit vorheriger Zustimmung des betreffenden Rechteinhabers oder auf der Grundlage einschlägiger gesetzlicher Erlaubnisvorschriften zulässig. 